\section{Targeting Wnt signaling in acute myeloid leukemia} stem cells

In this work we tested whether targeting Wingless and Int-1 (Wnt) signaling in acute myeloid leukemia (AML) leukemic stem cells (LSC) by using a porcupine inhibitor (WNT974) is a good strategy to eradicate LSC. Wnt/ $\beta$-catenin is an evolutionarily conserved pathway that is involved in embryonic development and stem cells by regulating cell fate and differentiation decisions. ${ }^{1}$ In normal conditions, the $\mathrm{Wnt} / \beta$-catenin pathway mediates normal hematopoietic stem cell self-renewal, proliferation and differentiation and is tightly controlled., ${ }^{2,3}$ While $\beta$-catenin is highly expressed in normal hematopoietic stem cells, it is downregulated during myeloid differentiation. ${ }^{2,4}$ Aberrant activation of this pathway gives rise to the accumulation of $\beta$-catenin in the nucleus, and promotes the transcription of many oncogenes such as $c-M y c$ (MYC). $\beta$-catenin is highly expressed in AML patients and overexpression of $\beta$-catenin in normal $\mathrm{CD}_{3} 4^{+}$ hematopoietic precursors in vitro leads to a myeloproliferative disorder of immature cells. ${ }^{5,6}$ Further work identified a crucial role of the $\mathrm{Wnt} / \beta$-catenin pathway in the development of LSC, ${ }^{7}$ and different authors proposed targeting the Wnt pathway in AML blasts. ${ }^{8-11}$ Thus, we

A

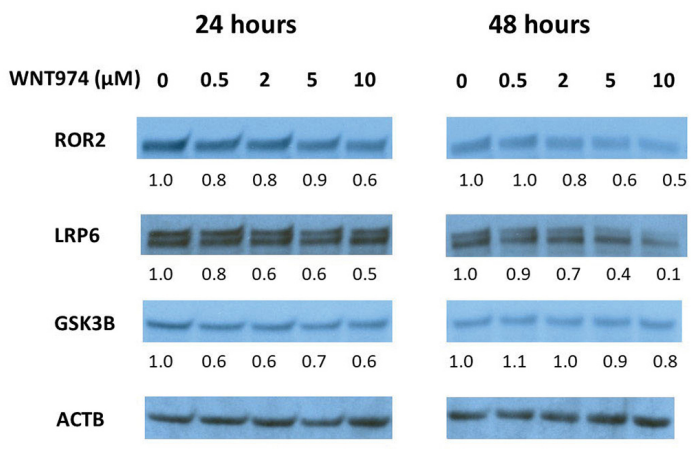

C

Luciferase assay

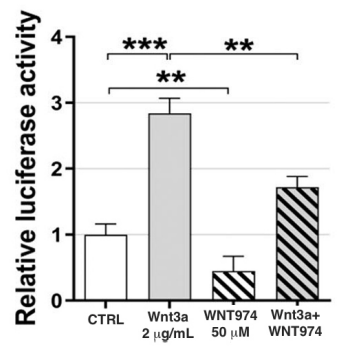

hypothesize that targeting Wnt in LSC, using a novel Wnt inhibitor called WNT974, could be an efficient strategy to eradicate LSC, prevent relapse and improve overall outcomes in AML.

WNT974 is an inhibitor of the enzyme porcupine, which is a membrane-bound O-acyltransferase located in the endoplasmic reticulum, and it is required for the palmitoylation of Wnt ligands. Inhibition of porcupine prevents the secretion and activity of Wnt ligands outside the cell, leading to a decrease in Wnt ligand cell surface receptor phosphorylation and a reduction in the expression of Wnt target genes. ${ }^{12,13}$ Several studies documented that WNT974 is able to target the Wnt pathway in solid tumors and the LSC in chronic myeloid leukemia. ${ }^{14}$ Based on this information, we hypothesized that Wnt inhibition by using WNT974 may block the aberrant Wnt activation in AML LSC and prevent leukemogenesis. To verify this hypothesis, we first evaluated whether WNT974 treatment can inhibit the Wnt signaling pathway using the K562 cell line, which is the cell line used initially to report the activity of this drug in myeloid leukemias.

Treatment of K562 cells with dimethylsulfoxide (DMSO), as a control, or WNT974 at different concentrations $(0.5,2.5$ and $10 \mu \mathrm{M})$ for 24 and $48 \mathrm{~h}$ showed a reduction of Wnt pathway targets such as ROR2, LRP6 and GSK3B protein (Figure 1A), and AXIN2 mRNA

B

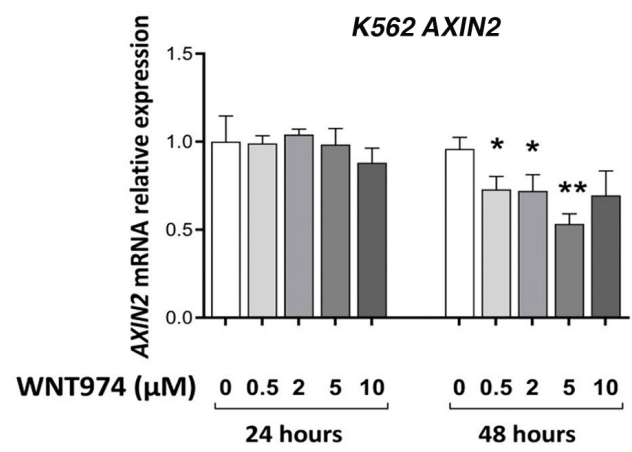

D

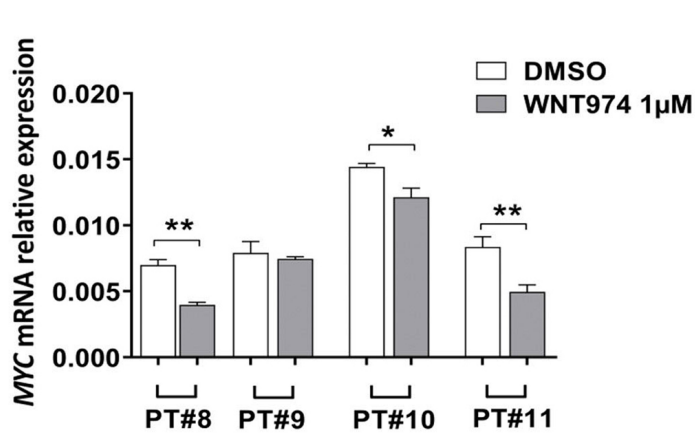

Figure 1. Validation of WNT974 activity in cell lines. (A) Western blot assay of ROR2, LRP6 and GSK3B after WNT974 treatment or control (dimethylsulfoxide) for 24 and $48 \mathrm{~h}$. Densitometry analysis was conducted using ImageJ software. Band intensity is reported relative to $\beta$-actin (ACTB). (B) AXIN2 mRNA fold change expression of K562 cells treated with WNT974 at different concentrations, or control (DMSO), for $24 \mathrm{~h}$. * $P<0.05$, **P<0.01 (t-test). (C) Luciferase Renilla assay of HCT116 cells transfected with a wild-type or mutant $\beta$-catenin activity reporter vector. Thirty hours after transfection, the cells were treated with Wnt3a ligand $(200 \mathrm{ng} / \mathrm{mL})$, WNT974 $5 \mu \mathrm{M}$, or a combination for $24 \mathrm{~h}$. Data are normalized to the control wild-type. $* P<0.05, * * P<0.01, * * * P<0.001$ ( $t$-test). (D) $M Y C$ mRNA relative expression of bone marrow CD34 ${ }^{+}$selected primary acute myeloid leukemia cells treated with WNT974 $1 \mu \mathrm{M}$ for $24 \mathrm{~h}(* P<0.05$, $* * P<0.01$ by the $t$ test). GAPDH was used as a normalizer. 
A

PT \#2

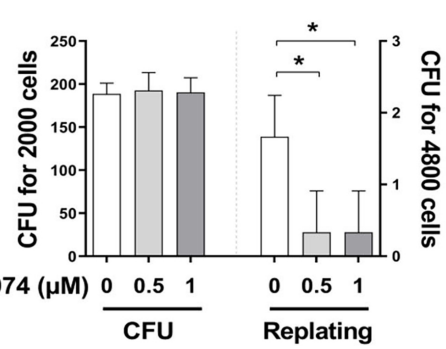

B

CD34 ${ }^{+}$Bone marrow

\#1

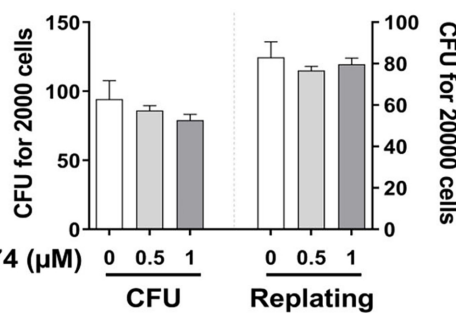

PT \#4

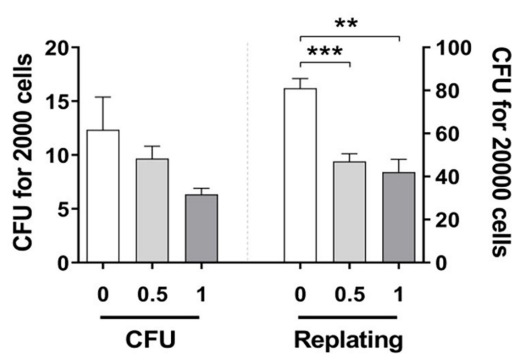

CD34 ${ }^{+}$Bone marrow

\#2

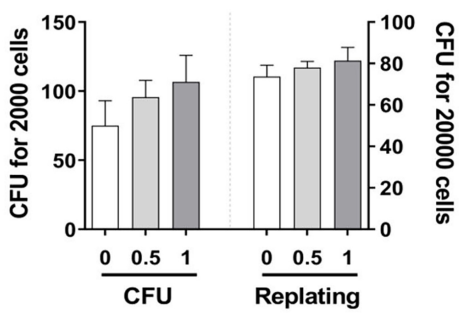

PT \#5

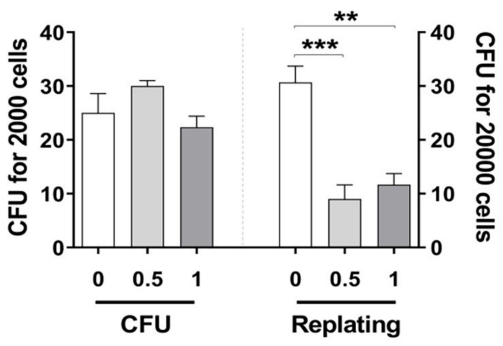

CD34 ${ }^{+}$Bone marrow

\#3

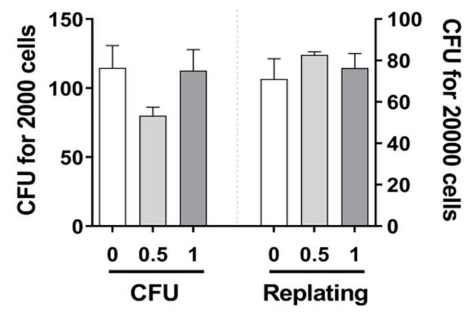

C PT\#1

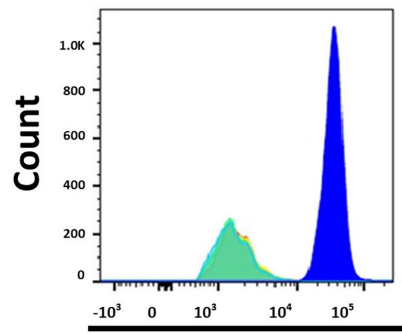

D

PT\#2

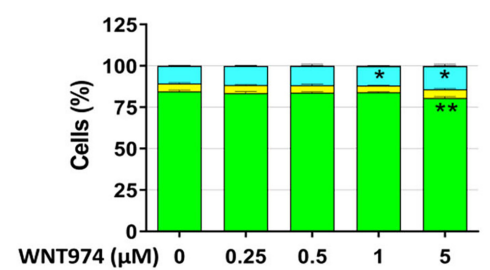

Pacific Blue
PT\#3

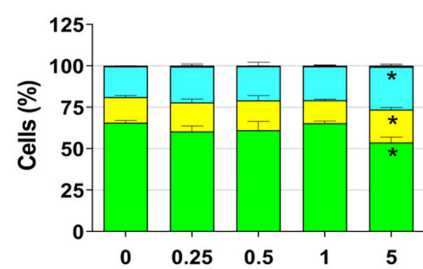

PT\#2

PT\#3
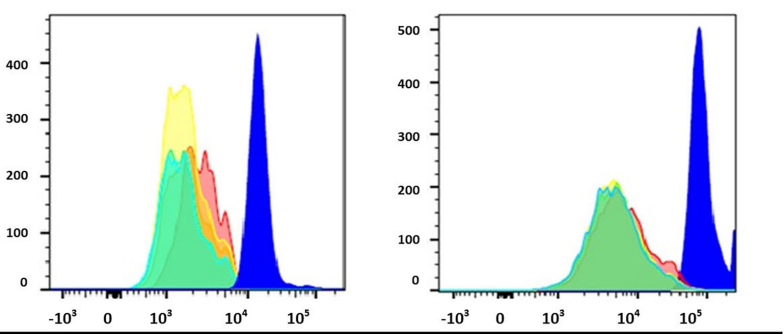

Day O_CTRL

$\square$ CTRL DMSO 0.1\%

WNT974 $0.25 \mu \mathrm{M}$

$\square$ WNT974 $0.5 \mu \mathrm{M}$

WNT974 $1 \mu \mathrm{M}$

WNT974 $5 \mu \mathrm{M}$

Figure 2. WNT974 treatment of primary CD34+ acute myeloid leukemia patients' samples decreased self-renewal but not apoptosis or quiescence. (A) Colonyforming unit (CFU) assay of $\mathrm{CD} 34^{+}$primary acute myeloid leukemia (AML) cells treated with WNT974 drug at different concentrations or vehicle (dimethylsulfoxide, DMSO 0.1\% volume). After 2 weeks, colonies were scored and replated. A second scoring was done 2 weeks after replating. The graph shows mean and standard deviation values from a technical triplicate. $* P<0.05, * * P<0.01, * * * P<0.001$ (t-test). (B) CFU assay of CD34 ${ }^{+}$cells from bone marrow of adult healthy donors treated with WNT974 drug at different concentrations or vehicle (DMSO 0.1\% volume). After 2 weeks, colonies were scored and replated. A second scoring was done 2 weeks after replating. All tests (t-tests) were non-significant. (C) CellTrace Violet assay of three CD34 ${ }^{+}$selected primary AML cells treated with WNT974 at various concentrations, or vehicle (DMSO 0.1\%). 7-AAD staining was conducted to select only the living cells. The graphs show the different counts at day 0 (dark blue, only control), and at day 6 (3 days of incubation, plus 3 days of treatment or vehicle). No values are statistically significant except for that for patient 2 at the higher dose of WNT974 ( $P=0.0077, t$-test). (D) Annexin V-FITC and propidium iodide (PI) analysis by flow cytometry of CD34 ${ }^{+}$selected primary AML cells treated with WNT974 or control at different concentrations for $72 \mathrm{~h}$. Living (Annexin $\left.\mathrm{PI}^{-}\right)$, Early apoptosis (Annexin $\left.{ }^{+}, \mathrm{Pl}^{-}\right)$, Late apoptosis $\left(\mathrm{Annexin}{ }^{+}\right.$, $\mathrm{Pl}^{+}$) and Necrosis $\left(\right.$Annexin $-\mathrm{Pl}^{+}$). Each analysis was conducted in technical triplicates. $* P<0.05, * * P<0.01$ ( $t$-test). 
expression (Figure 1B). We also confirmed that the drug efficiently targets the Wnt pathway by using a luciferase reporter vector in HCT116, a colorectal cancer cell line that shows constitutive activation of the Wnt pathway (Figure 1C). We also confirmed Wnt targeting by WNT974 in primary AML samples (Online Supplemental Table S1 for patients' details). As shown in Figure 1D, WNT974 decreased mRNA expression of the MYC gene, which is considered one of the main oncogenes driven by abnormal Wnt/ $\beta$-catenin signaling. We also observed a similar reduction in mRNA expression of other Wnt target genes such as CTNNB1 and GSK3B after WNT974 treatment of primary AML samples (Online Supplementary Figure S1A).

Next, we wanted to investigate whether the treatment was able to affect LSC functions in primary AML patients' samples. To assess whether targeting the Wnt pathway affects leukemia cell self-renewal, bone marrow

\section{A}

AML murine \#1

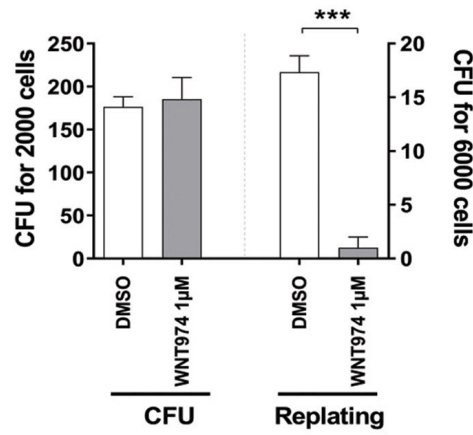

B

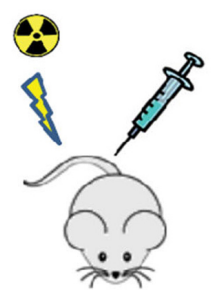

$1^{\text {st }}$ Recipient
AML murine \#2

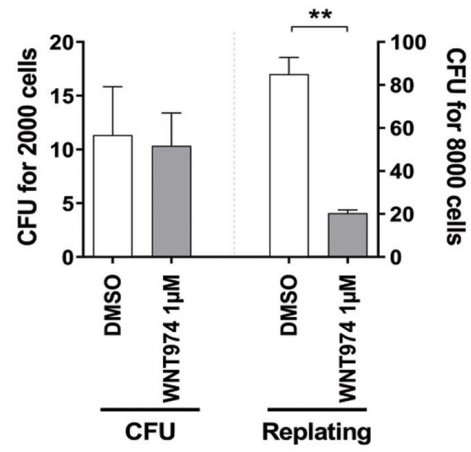

Treatment 1 week $5 \mathrm{mg} / \mathrm{Kg} /$ day
$5 * 10^{6} \mathrm{AML}+$

$5 * 10^{5}$ Healthy BM

5 weeks

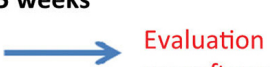

engraftment

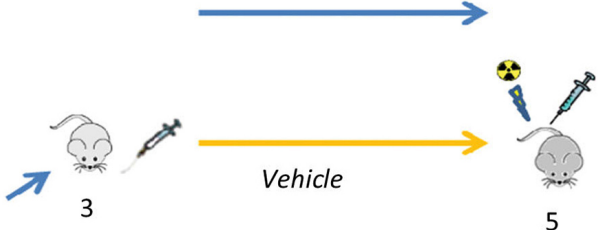

$5 * 10^{6} \mathrm{AML}+$

$5 * 10^{5}$ Healthy BM

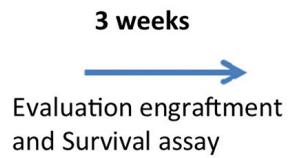

$5 * 10^{6} \mathrm{AML}+$

$5 * 10^{5}$ Healthy BM

\section{$2^{\text {nd }}$ Recipient}

\section{Survival of secondary transplanted mice}

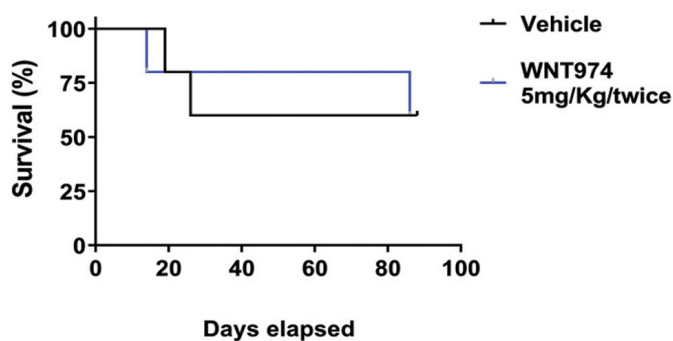

D
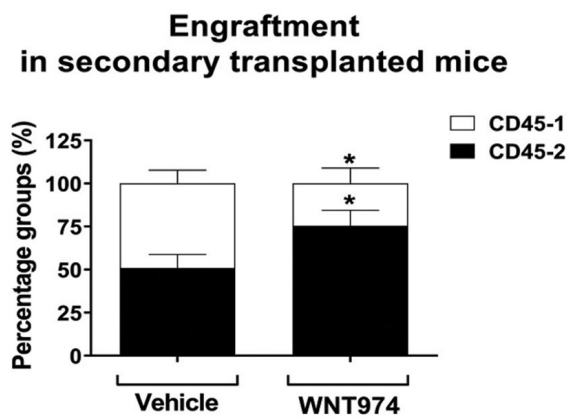

Figure 3. WNT974 activity in a primary murine model of acute myeloid leukemia. (A) Colony-forming unit assay of $\mathrm{CD} 117^{+} \mathrm{m} \mathrm{MIITD/WT} / \mathrm{Flt3} 3^{I T D / W T}$ bone marrow cells treated with WNT974 at 0.5 and $1 \mu \mathrm{M}$ or vehicle (dimethylsulfoxide, DMSO $0.1 \%$ ) for 2 weeks. Results from the first scoring are shown in the left panel. After scoring, the cells were replated in fresh Methocult media and scored after another 2 weeks (right panel). Student $t$-test analysis: $* P<0.05$, $* * P<0.01$ and $* * * P<0.001$. (B) Flow chart of the in vivo re-transplantations experiment. (C) Overall survival of secondary transplanted mice ( $\mathrm{n}=5$ mice per group). (D) Evaluation of engraftment at 1 month. ${ }^{*} P<0.05$ (t-test). 
CD34+-selected cells from five AML patients were treated with WNT974 or the control (DMSO) and used in colony-forming unit (CFU) assays. We found no significant differences in the number of colonies in primary CFU after 2 weeks of culture (Figure 2A, Online Supplementary Figure $S 1 B$, left panels). Primary colonies were harvested and then re-plated in methylcellulose for an additional 14 days. We found significant decreases in the number of secondary colonies in WNT974-treated cells compared with cells treated with the control, suggesting that WNT974 targeting has an impact on LSC self-renewal (Figure 2A, Online Supplementary Figure S1A, right panels). We performed the same experiment using bone marrow from three adult healthy donors and CD34 ${ }^{+}$cells from three cord blood specimens. As shown in Figure 2B and Online Supplementary Figure S1C, the treatment did not affect normal cells.

Next, we analyzed whether WNT974 has a role in regulating LSC quiescence by using cell membrane labeling retention assays. For these experiments, CD34 ${ }^{+}$-selected samples from three AML patients were stained with cell trace violet (CTV) dye, which incorporates into the cell membrane and can be detected by flow cytometry. CD34 ${ }^{+}$-selected AML patients' cells were isolated, labeled with CTV, and treated for 3 days with WNT974 and control. The number of viable $\left(7-\mathrm{AAD}^{\text {neg }}\right)$, quiescent $\left(\mathrm{CTV}^{\text {high }} / \mathrm{CD} 34^{+}\right)$cells was then determined by flow cytometry. CTV high/CD34+ cells have been shown previously to increase leukemia-initiating ability, and enhanced engraftment in NSG mice. However, in all analyzed patients' samples, we did not find a significant decrease in the number of CTV $\mathrm{Cigh}^{\mathrm{hi}} / \mathrm{CD} 34^{+}$cells after treatment with WNT974, compared with controls, except for patient 2 at the highest WNT974 dose $(t$-test; $P=0.0077)$ (Figure 2C). In order to assess whether the treatment can affect the bulk cell population, we performed an annexin-V/propidium iodide assay on the same CD34+ AML patients' samples treated with WNT974 after $72 \mathrm{~h}$, and found only a slightly increase of apoptosis at the higher concentration (Figure 2D).

Finally, we investigated whether WNT974 could also effectively target LSC in vivo. To test the effects of WNT974 in vivo, we used our well-characterized $M l l^{\mathrm{PTD} / \mathrm{WT}} /$ Flt $3^{\mathrm{ITD} / \mathrm{WT}}$ double knock-in CN-AML mouse model. ${ }^{15}$ This model develops an aggressive AML with $100 \%$ penetrance and leads to death within 5 to 8 weeks in secondary bone marrow transplantation. ${ }^{15}$ First, we confirmed that WNT974 treatment has a similar effect on the primary murine AML cells in this model by conducting a CFU in vitro assay on $\mathrm{CD} 117^{+}$murine cells from bone marrow of AML mice. We observed that the treatment was able to reduce proliferation after the re-plating (Figure 3A) similar to the treatment in human AML cells. Next, we transplanted $M l^{\text {PTD/WT }} / F l t 3^{\mathrm{ITD} / \mathrm{WT}}$ leukemic cells $\left(\mathrm{CD} 45.2^{+}\right.$) into lethally irradiated wild-type (WT)-BoyJ $\left(\mathrm{CD} 45.1^{+}\right)$mice together with whole bone marrow cells from WT-BoyJ donors $\left(C D 45.1^{+}\right)$. Two weeks after engraftment, mice were treated with WNT974 or vehicle (control) at a dose of $5 \mathrm{mg} / \mathrm{kg}$ twice a day by oral gavage for 1 week. The design of the experiment is shown in Figure $3 \mathrm{~B}$. After the last dose, mice were sacrificed and bone marrow and spleen cells were obtained. We measured the Wnt target gene $M Y C$ as a surrogate for Wnt pathway targeting in leukemic cells in vivo. We found significant downregulation of murine Myc in spleen cells that were used during the primary transplants (Online Supplementary Figure S2A). Next, we determined whether Wnt pathway knock-down by WNT974 affected leukemia engraftment in secondary bone marrow trans- plantation, a key feature of stem cells. Leukemic donor cells obtained from mice spleen treated with WNT974 or vehicle were transplanted into lethally irradiated BoyJ recipients. Despite observing a reduction of Wnt pathway target gene expression in the spleens of secondary transplanted mice (Online Supplementary Figures S2B-E), we did not observe any differences between overall survival (Figure 3C), or engraftment at 1 month (Figure 3D), after re-transplantation. In conclusion, the data show that WNT974 treatment was able to reduce Wnt pathway activity in leukemic cells and decreased self-renewal of primary AML LSC in vitro. However, WNT974 treatment alone was unable to impact LSC functions in vivo. For these experiments, we used high doses of WNT974, higher than the equivalent doses used in human clinical trials with this drug. Thus, it is unlikely that the lack of in vivo effect is due to poor pharmacokinetics or pharmacodynamics. This is supported by the fact that we observed Wnt target downregulation in leukemic cells in the treated mice. Another possibility is that other pathways or even Wnt reactivation could compensate for the initial Wnt downregulation in LSC, and this could be driven by the microenvironment. It is likely that a combination of WNT974 with other active agents in AML could lead to sustained targeting and eradication of LSC in AML as was shown in chronic myeloid leukemia. ${ }^{14}$

Felice Pepe, ${ }^{1}$ Marius Bill, ${ }^{1}$ Dimitrios Papaioannou, Malith Karunasiri, ${ }^{1}$ Allison Walker, ${ }^{1}$ Eric Naumann, ${ }^{1}$

Katiri Snyder, ${ }^{1}$ Parvathi Ranganathan, ${ }^{1,2}$

Adrienne Dorrance ${ }^{1,2}$ and Ramiro Garzon ${ }^{1,2}$

${ }^{1}$ The Ohio State University Comprehensive Cancer Center and ${ }^{2}$ Division of Hematology, Department of Internal Medicine, The Ohio State University, Columbus, OH, USA

Correspondence:

RAMIRO GARZON - ramiro.garzon@osumc.edu.

doi:10.3324/haematol.2020.266155

Received: July 7, 2020

Accepted: September 8, 2021.

Pre-published: September 16, 2021.

Disclosures: no conflicts of interest to disclose.

Contributions: $R G$ conceived the idea; $R G$ and FP designed the study; $F P, M K$ and $E N$ performed the in vitro experiments; $F P, M B$ and $K S$ performed the in vivo experiments; $F P, D P, P R, A D$ and $R G$ analyzed and interpreted the data; $R G$ supervised the study; $F P, A D$ and $R G$ drafted the manuscript.

\section{References}

1. Steinhart Z, Angers S. Wnt signaling in development and tissue homeostasis. Development. 2018;145(11):dev146589.

2. Luis TC, Ichii M, Brugman MH, Kincade P, Staal FJT. Wnt signaling strength regulates normal hematopoiesis and its deregulation is involved in leukemia development. Leukemia. 2012;26(3):414-421.

3. Staal FJ, Sen JM. The canonical Wnt signaling pathway plays an important role in lymphopoiesis and hematopoiesis. Eur J Immunol. 2008; 38(7):1788-1794.

4. Luis TC, Naber BAE, Roozen PPC, et al. Canonical wnt signaling regulates hematopoiesis in a dosage-dependent fashion. Cell Stem Cell. 2011;9(4):345-356.

5. Staal FJ, Famili F, Garcia Perez L, Pike-Overzet K. Aberrant Wnt Signaling in Leukemia. Cancers (Basel). 2016;8(9):78.

6. Ashihara E, Takada T, Maekawa T. Targeting the canonical Wnt/beta-catenin pathway in hematological malignancies. Cancer Sci. 2015;106(6):665-671.

7. Wang Y, Krivtsov AV, Sinha AU, et al. The Wnt/beta-catenin pathway is required for the development of leukemia stem cells in AML. Science. 2010;327(5973):1650-1653.

8. Minke KS, Staib P, Puetter A, et al. Small molecule inhibitors of WNT signaling effectively induce apoptosis in acute myeloid leukemia cells. Eur J Haematol. 2009;82(3):165-175.

9. Fiskus W, Sharma S, Saha S, et al. Pre-clinical efficacy of combined 
therapy with novel $\beta$-catenin antagonist BC2059 and histone deacetylase inhibitor against AML cells. Leukemia. 2015;29(6):12671278.

10. Griffiths EA, Golding MC, Srivastava P, et al. Pharmacological targeting of $\beta$-catenin in normal karyotype acute myeloid leukemia blasts. Haematologica. 2015;100(2):e49-e52.

11. Jiang X, Mak PY, Mu H, et al. Disruption of Wnt/ $\beta$-catenin exerts antileukemia activity and synergizes with FLT3 inhibition in FLT3 mutant acute myeloid leukemia. Clin Cancer Res. 2018;24(10):24172429.

12. Proffitt KD, Madan B, Ke Z, et al. Pharmacological inhibition of the Wnt acyltransferase PORCN prevents growth of WNT-driven mam- mary cancer. Cancer Res. 2013;73(2):502-507.

13. Liu J, Pan S, Hsieh MH, et al. Targeting Wnt-driven cancer through the inhibition of Porcupine by LGK974. Proc Natl Acad Sci U S A. 2013;110(50):20224-20229.

14. Agarwal P, , Zhang B, Ho Y, et al. Enhanced targeting of CML stem and progenitor cells by inhibition of porcupine acyltransferase in combination with TKI. Blood. 2017;129(8):1008-1020.

15. Zorko NA, Bernot KM, Whitman SP, et al. Mll partial tandem duplication and Flt3 internal tandem duplication in a double knock-in mouse recapitulates features of counterpart human acute myeloid leukemias. Blood. 2012; 120(5):1130-1136. 